\title{
Structure Improvement and Optimization of Spool Valve Throttling Grooves of the Multi- way valve
}

\author{
Liping $\mathrm{Xu}^{1, \mathrm{a}}$, Haoyi Ma ${ }^{1, \mathrm{~b}^{*}}$ and Liujin Cai ${ }^{1, \mathrm{c}}$ \\ ${ }^{1}$ Henan University of Science and Technology, School of Mechatronics Engineering, Henan, \\ Luoyang, 471000, China \\ axlpzz@163.com, b253279482@qq.com, cyycailj@163.com \\ ${ }^{*}$ Corresponding author
}

Keywords: Multi-way valve; Throttling grooves; Structure improvement; Particle swarm optimization algorithm; Flow characteristic

\begin{abstract}
Slide valve spool throttling grooves play a very important role for the working performance of multi-way valve. In order to solve some problems such as serious pressure loss and excessive operating force of hands, when multi-way valve is in working. An improved scheme of new type throttling grooves is provided, the mathematical model of new-type throttling grooves is constructed, particle swarm optimization algorithm is used to optimize the structure of throttling grooves and we can get the optimal design parameters. Thus, the flow characteristic of the valve port is improved, and the working performance of multi-way valve is promoted.
\end{abstract}

\section{Introduction}

Multiple directional control valve is abbreviated as multi-way valve, it's used to control the direction and flow of fluid in the hydraulic system, which has the function of both direction control valve and flow control valve. ${ }^{[1]}$ Throttling groove is mainly used in the slide valve spool of multi-way valve, and its flow characteristic determines the working performance of multi-way valve. The reasonable structure of throttling groove can reduce multi-way valve's reversing impact and vibration during working, and ensure the stability and comfort of actuators. ${ }^{[2]}$ Although we have more in-depth understanding of throttling groove, according to the traditional formula, flow characteristic of throttling grooves obtained by following valve port opening show a large and irregular change. Therefore, in order to improve valve port flow characteristic of multi-way valve, it is necessary to carry out the optimization research of throttling grooves structure.

\section{The Structure Improvement of New Type Throttling Grooves}

The Structure form of New Type Throttling Grooves. In order to solve some problems such as serious pressure loss and excessive operating force of hands, when multi-way valve is in working. An improved scheme of special form new type throttling grooves four-position spool valve is provided, which can improve flow characteristic of valve port and enhance working performance of multi-way valve. Compared with the $U$ throttling grooves in original multi-way valve, the triangular grooves and the top of the triangle with a hole are cut in the shoulder of four-position spool valve, which is abbreviated as new type throttling grooves, as shown in figure 1. 

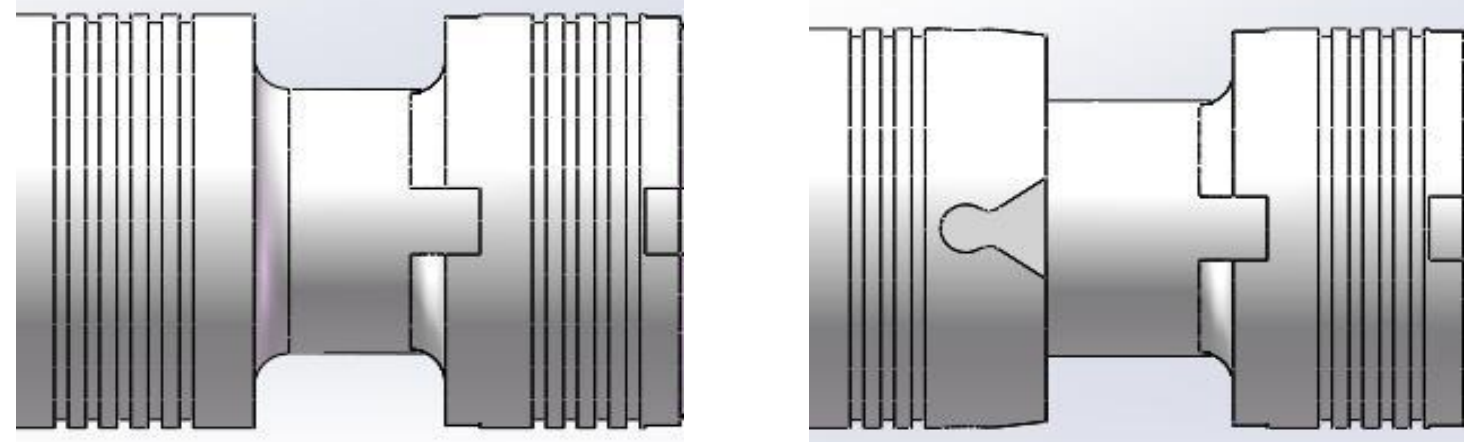

Figure 1 The structural sketch of original spool and new spool

The Flow Area Calculation of Throttling Grooves Spool Valve. The flow area calculation of the throttling grooves valve port is not only the basis of the numerical simulation of multi-way valve, but also has a guiding significance for improving the flow characteristic of valve port. The form of the multi-way valve port includes full opening and non full opening, it has a main influence on the flow control characteristic of the multi-way valve. The flow area calculation of full opening is very simple, so it's easy to calculate. However, the valve port of non full opening has a good throttling effect, and it is a common valve port form. But, there is a complex nonlinear relationship between the flow area and valve port opening, it's difficult to obtain accurate flow area mathematical expression in most cases.

The structural parameter diagram of $U$ throttling grooves, as shown in figure 2.
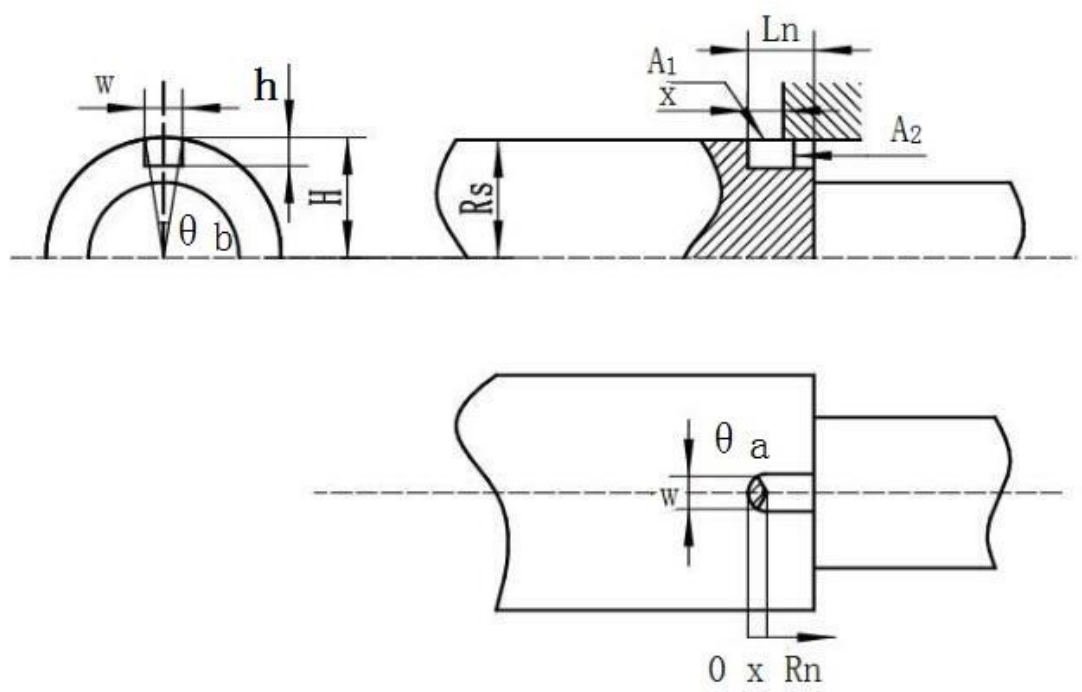

Figure 2 The computation diagram of $U$ throttling grooves flow area

The formula of flow area is deduced as follows:

when the valve opening is $0<x \leq R_{\mathrm{n}}$ :

$$
\begin{gathered}
W=2 \sqrt{R_{\mathrm{n}}^{2}-\left(R_{\mathrm{n}}-x\right)^{2}} \\
H=\sqrt{R_{\mathrm{s}}^{2}-(W / 2)^{2}} \\
A_{1}=\int_{0}^{x} \theta_{b} R_{\mathrm{s}} d x \\
A_{2}=\frac{R_{\mathrm{s}}^{2} \theta_{\mathrm{b}}}{2}-\frac{W H}{2}+W h
\end{gathered}
$$

when the valve opening is $R_{\mathrm{n}}<x \leq L_{\mathrm{n}}$ : 


$$
\begin{gathered}
W=2 R_{\mathrm{n}} \\
H=\sqrt{R_{\mathrm{s}}^{2}-(W / 2)^{2}}, \quad \theta_{\mathrm{b}}=2 \arctan \frac{W}{2 H} \\
A_{1}=\frac{1}{2} \pi R_{\mathrm{n}}^{2}+\int_{R_{\mathrm{n}}}^{L_{\mathrm{n}}} \theta_{\mathrm{b}} R_{\mathrm{s}} d x \\
A_{2}=\frac{R_{\mathrm{s}}^{2} \theta_{\mathrm{b}}}{2}-\frac{W H}{2}+W h
\end{gathered}
$$

The structural parameter diagram of new type throttling grooves, as shown in figure 3.
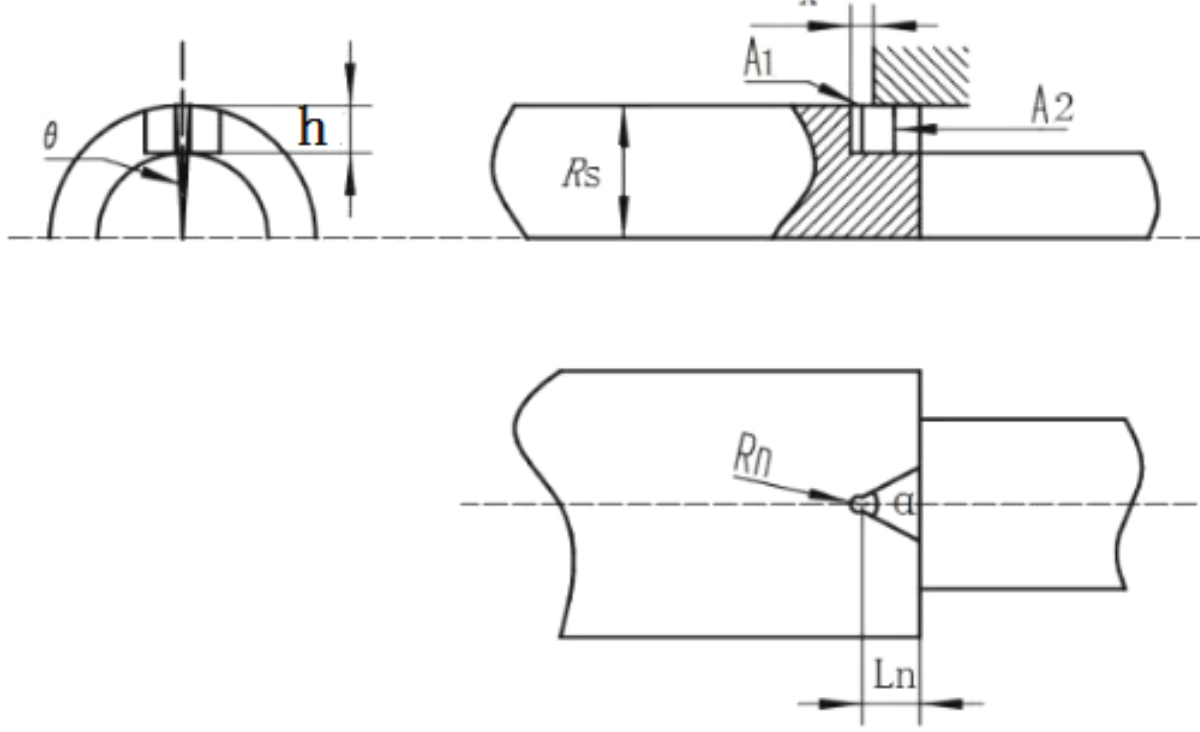

Figure 3 The computation diagram of new throttling grooves flow area

The formula of flow area is deduced as follows:

when the valve opening is $0<x \leq R_{\mathrm{n}}+R_{\mathrm{n}} \cos \frac{\alpha}{2}$ :

$$
\begin{gathered}
A_{1}=\int_{0}^{x} \theta R_{\mathrm{s}} d x \\
A_{2}=\theta R_{\mathrm{s}} h \\
\theta=2 \arcsin \frac{R_{\mathrm{n}}}{R_{\mathrm{s}}}
\end{gathered}
$$

when the valve opening is $R_{\mathrm{n}}+R_{\mathrm{n}} \cos \frac{\alpha}{2}<x \leq L_{\mathrm{n}}-R_{\mathrm{n}} \cos \frac{\alpha}{2}$ :

$$
\begin{gathered}
A_{1}=\frac{(360-\alpha)}{360} \pi R_{\mathrm{n}}^{2}+\left(x-R_{\mathrm{n}}\right)^{2} \tan \frac{\alpha}{2} \\
A_{2}=2\left(x-R_{\mathrm{n}}\right) \tan \frac{\alpha}{2} \cdot h
\end{gathered}
$$

\section{Optimization of the New Section of the Groove Structure}

The intelligent optimization algorithm is mainly used to optimize the structure of the throttling groove, which includes genetic algorithm, particle swarm optimization algorithm. Relative to genetic algorithm, particle swarm algorithm retains the global searching strategy based on population, without needing complex encoding and genetic operation. Because of its few parameters, easy convergence and optimization, so it has been widely used in structural optimization design. 
When the actuators operate in constant pressure difference, the optimization design of throttling grooves is to obtain corresponding structure parameters, according to the requirements of valve port opening-flow characteristics. Then the suitable design variables are obtained through using particle swarm algorithm to optimize the throttling grooves structure. In order to maintain the stability of hydraulic system in practical working, spool valve throttling grooves also work basically in constant pressure difference, so this paper is also doing research in constant pressure difference.

The Optimization Principle of Throttling Grooves. According to the requirements of particle swarm optimization algorithm, first, the structural design variable space $R$ is defined, then a plurality of particles are initialized and particle's population is formed, and each particle corresponds to a feasible solution in the variable space, so each particle also has a value in objective function. The objective function value is better, the feasible solution is better. Finally, all particles will move in the variable space, the optimal solution of particle itself $P_{\text {best }}$ and the optimal solution of particle population $g_{\text {best }}$ determine particle's velocity, which influence the direction and distance of next step for the particle movement directly. Particle population's motion is equivalent to algorithm's iteration, and the particle movement rule is the iterative rule, the formula is expressed as: $D$-dimensional variable, population size is $n$, the position vector and velocity vector of the $i$ particle in the dimension variable space is $x_{i}=\left(x_{i 1}, x_{i 2}, \ldots, x_{i D}\right)$ and $v_{i}=\left(v_{i 1}, v_{i 2}, \ldots v_{i D}\right)$ respectively, the optimal solution of the $i$ particle itself is $P_{\text {best }, i}=\left(p_{i 1}, p_{i 2}, \ldots, p_{i D}\right)$, and the optimal solution of particle populations is $\mathrm{g}_{\text {best }, i}=\left(g_{1}, g_{2}, \ldots, g_{D}\right)$. The iterative formula for the velocity and position of particles in $k+1$ dimensions

$$
\begin{gathered}
v_{i, d}^{(k+1)}=\omega v_{i, d}^{(k)}+c_{1} \xi\left(p_{i, d}^{(k)}-x_{i, d}^{(k)}\right)+c_{2} \eta\left(g_{i, d}^{(k)}-x_{i, d}^{(k)}\right) \\
x_{i, d}^{(k+1)}=x_{i, d}^{(k)}+r v_{i, d}^{(k+1)} \\
i=1,2,3 \ldots n ; d=1,2,3 \ldots D
\end{gathered}
$$

In the formula, the velocity and position of particles is $v_{i, d}$ and $x_{i, d}$ respectively; the factor of $w$ maintaining original velocity is called inertia weight; $c_{1} 、 c_{2}$ are acceleration coefficient, $c_{1}$ has the ability to adjust the particle moving to its optimal position, which embodies the cognition of "particle"; $c_{2}$ has the ability to adjust the particle moving to the global optimal position of particle population, which embodies the cognition of "society"; $\xi, \eta$ are random numbers in the range of $[0,1]$; constraint factor is $r$; iteration times is $k$.

The Establishment of Optimization Model The structure parameters of new type throttling grooves are treated as design variables, the flow of fluid simulation during working is taken as particle optimization criteria, and the optimization mathematical model is established, when the four-position spool valve throttling grooves structure of multi-way valve is optimized. Therefore, in order to meet the requirements of the flow change of multi-way valve port, the sum of variance of the flow of simulation value and theoretical value under different valve port openings is regarded as the fitness function for the optimization mathematical model. In other words, the fitness function is objective function:

$$
\min (s)=\frac{1}{m} \sum_{j=1}^{m}\left(q_{j}-f_{j}(X)\right)^{2}
$$

In the formula, $m$ is the number of different valve port openings; $q_{j}$ is the flow under $j$ valve port opening, that is the flow of simulation value; $f_{j}(X)$ is the flow of theoretical value under $\mathrm{j}$ is valve port opening; $X=\left\{\alpha, R_{\mathrm{n}}, L_{\mathrm{n}}, h\right\}^{\mathrm{T}}$ is design variables; $s$ is variance. 


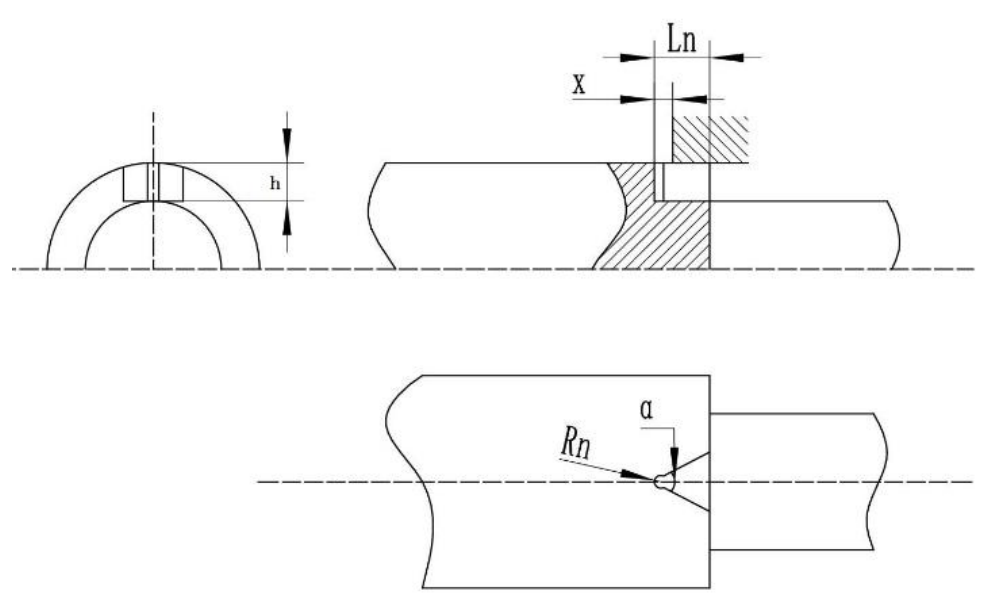

Figure 4 The design parameter of new throttling grooves

When the objective function has minimum value, the corresponding feasible solution $X$ is the optimal solution to satisfy the conditions, $D$-dimensional variable determine the optimal solution's dimension. Constraints: (1) Within the fluid flow field simulation of multi-way valve, the inlet flow and the working load pressure are constant; (2) The number of throttling grooves is constant, in order to eliminate the effect of circumferential unbalanced force, the number of throttling grooves is 4; (3) The depth of the throttling grooves is a fixed value, $h=4.5 \mathrm{~mm} . q_{\mathrm{j}}$ and $f_{j}(X)$ are a function of flow area as a variable individually, however, the flow area is a function of $X=\left\{\alpha, R_{\mathrm{n}}, L_{\mathrm{n}}, h\right\}^{\mathrm{T}}$. The design parameter of new type throttling grooves are shown in figure 4 . So the objective function of $\min (\mathrm{s})$ depends on the design parameter of new type throttling grooves.

The Process of Optimization Design. When the throttling grooves structure of multi-way valve is optimizing, the design variables of mathematical optimized model are treated as particle individuals in the population, then the structural parameters are optimized, four groups of simulation experiment samples are designed, as shown in table 1, the flow of fluid field simulation value of operating valve port is taken as the evaluation goals. Finally, through mathematical analysis and particle swarm optimization algorithm, we can solve the problem of structure design of valve port throttling grooves conversely.

Tab 1 The experimental samples of fluid simulation

\begin{tabular}{cllll}
\hline Sample & $\alpha /{ }^{\circ}$ & $R_{\mathrm{n}} / \mathrm{mm}$ & $L_{\mathrm{n}} / \mathrm{mm}$ & $h / \mathrm{mm}$ \\
\hline 1 & 60 & 2.5 & 4.8 & 4.5 \\
2 & 70 & 2 & 4.5 & 4.5 \\
3 & 80 & 1.5 & 4 & 4.5 \\
4 & 90 & 1 & 3.5 & 4.5 \\
\hline
\end{tabular}

Population size or the total particle number $n$ is $40, D$-dimensional variable is 4 , inertia weight $w$ is $0.7, c_{1} 、 c_{2}$ are acceleration coefficient $c_{1}=c_{2}=2$, iteration times $k$ is 100 , the maximum and minimum values are 0.5 and -0.5 respectively. When the algorithm runs, particles will follow the particle movement rule to move towards the optimal solution. Figure 5 shows the distribution of all the objective function value in the initial particle population, figure $6 \sim$ figure 8 shows the distribution of the objective function value of all the particles after several iterations. When the iteration is completed, particle swarm optimization algorithm is also over, the objective function value tends to steady distribution, and the population concentrate in the vicinity of the optimal solution basically, then the corresponding fitness function variables is the optimal design variables of new type throttling grooves structure. 


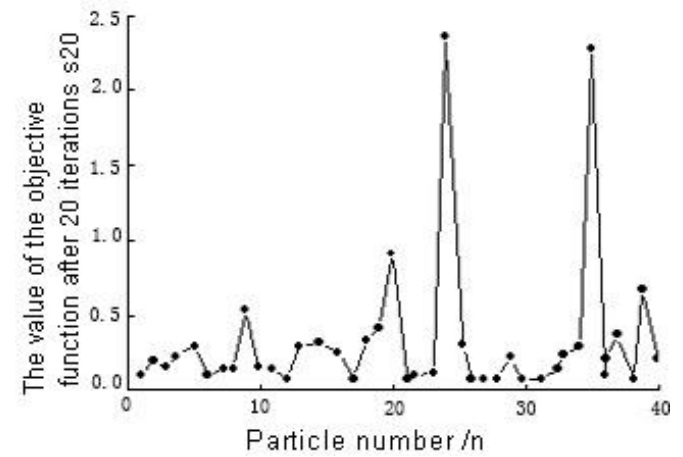

Figure 5 The distribution of initial population particles

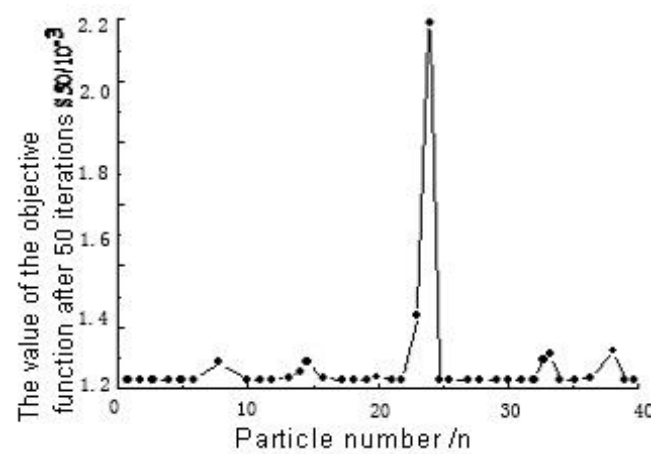

Figure 7 The distribution of particles in 50 iterations

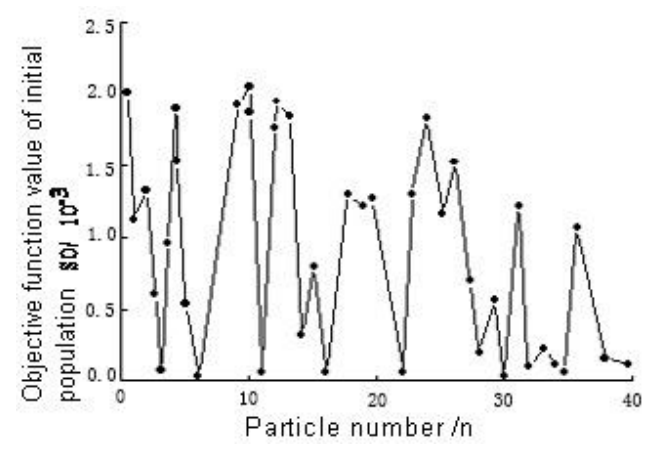

Figure 6 The distribution of particles in 20 iterations

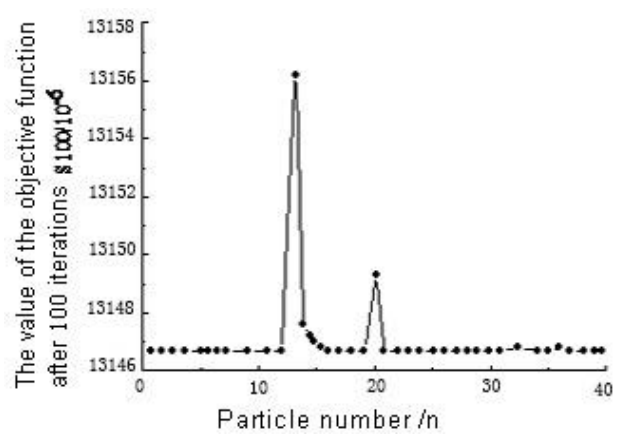

Figure 8 The distribution of particles in 100 iterations

The Optimization Design Results. The minimum value of the objective function $1.318687 \times 10^{-2}$ can be obtained when iteration is completed. Through optimizing design of four groups experiment samples and theoretical calculation, the corresponding design value of each sample will be obtained. Finally, the optimal structure parameters of new type throttling grooves is $\alpha=68^{\circ}, R_{\mathrm{n}}=1.473680 \times 10^{0} \mathrm{~mm}, L_{\mathrm{n}}=4.663471 \times 10^{0} \mathrm{~mm}, h=4.5 \mathrm{~mm}$, through numerical comparison combined with the simulation curve. In order to machine easily, the structure parameters obtained by particle swarm optimization algorithm should be rounded $\alpha=68^{\circ}$, $R_{\mathrm{n}}=1.5 \mathrm{~mm}, L_{\mathrm{n}}=4.7 \mathrm{~mm}, h=4.5 \mathrm{~mm}$.

The calculation program of valve port flow area with optimal design variables of throttling grooves structure will run in MathCAD software, which can calculate the valve port flow area rapidly. Two kinds of valve port flow area curves are shown in figure10, we can see that the flow area of new type throttling grooves is larger than original valve port flow area of $U$ throttling grooves under the same valve port opening. When multi-way valve achieve rated flow, the valve port opening of new valve will reduce, and flow regulation rage will increase. 


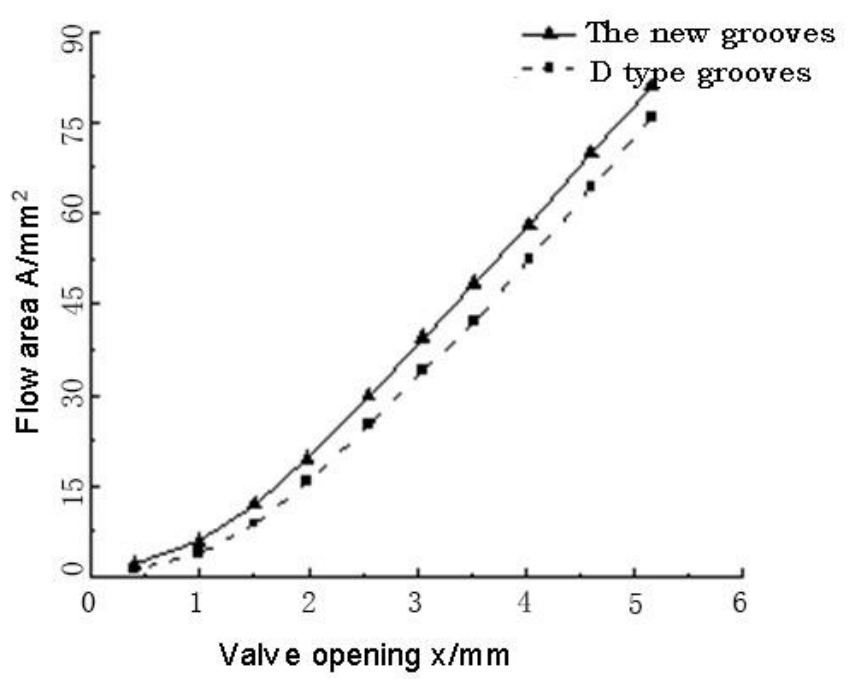

Figure 9 Two kinds of valve port flow area

The Verification of Flow Characteristic of Valve Port Opening. According to the optimal structure dimension parameters of the new type throttling grooves $\alpha=68^{\circ}, R_{\mathrm{n}}=1.5 \mathrm{~mm}$, $L_{\mathrm{n}}=4.7 \mathrm{~mm}, h=4.5 \mathrm{~mm}$ obtained by particle swarm algorithm, the fluid of multi-way valve is simulated by fluent on the basis of computational fluid dynamics theory and the optimal structure parameters, which can verify the simulation value and design value of valve port opening flow characteristic, as shown in figure 10 11. As can be seen from the figure, the trend of valve port flow rate change of optimization design value is a high coincidence with the flow of simulation value. At the same time, we can also see a good linearity between the flow and the valve port opening, especially before the opening of $2.5 \mathrm{~mm}$, the linear relationship obeys the rule of lineal change strictly, then with valve port opening increasing, the flow rate gain will decreases but still obeys linear change, this is all due to the role of new type throttling grooves.

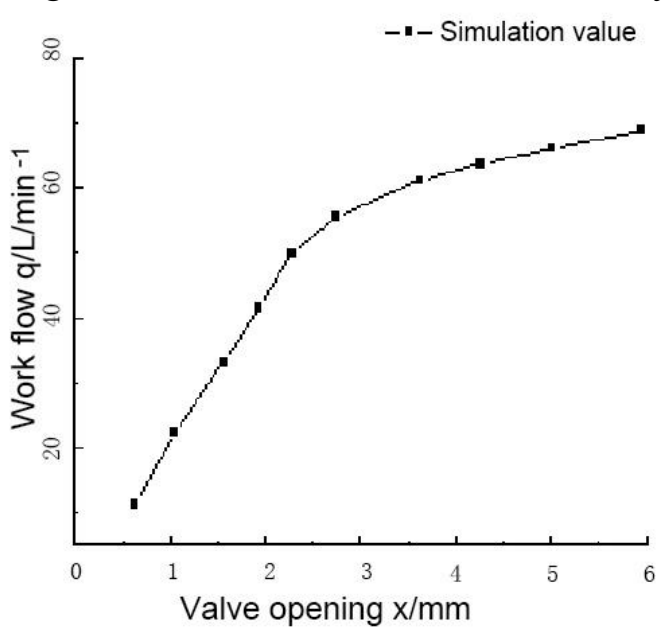

Figure 10 The valve port flow characteristic curve in simulation

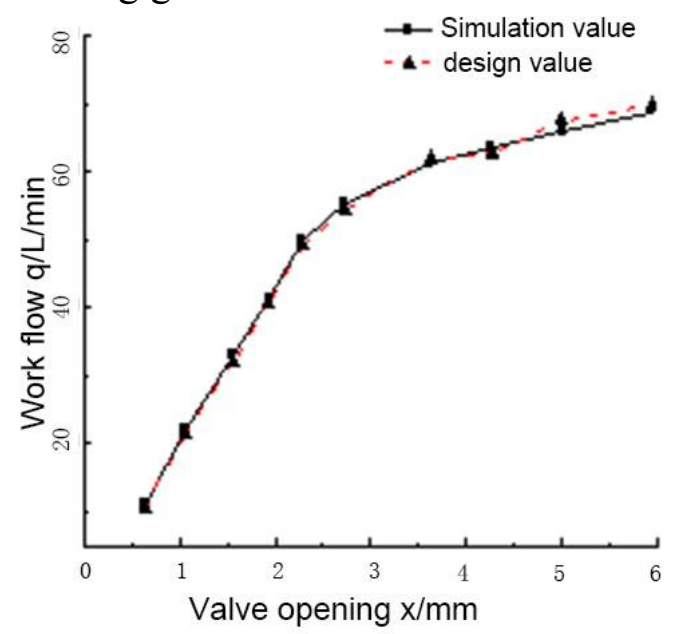

Figure 11 The valve port flow characteristic in simulation and design

\section{Conclusion}

In order to improve the multi-way valve port flow characteristic, the structure improvement scheme of new type throttling grooves is put forward, and the flow area of valve port is calculated with numerical analysis, the new type throttling grooves structure is optimized by using particle swarm optimization algorithm, and the optimal structural parameters are obtained.

1). With the same valve port opening, the flow area of new type throttling grooves is lager than original valve's, which can increase the regulation range of flow.

2). There is a good linear relationship between working flow and valve port opening after the 
new type throttling grooves of multi-way valve optimized, this results show the improved structure is reasonable and meet the requirements of valve port flow characteristic.

3). Optimization mathematical model of multi-way is established, and the new type throttling grooves of multi-way valve is optimized by using particle swarm algorithm. So the research method presented in this paper can be used as reference for the design of other hydraulic components.

\section{Acknowledgements}

The authors gratefully acknowledge natural science foundation of henan university of science and technology for financial support of this research work.(2015QN017)

\section{References}

[1] Li Zhuangyun. Hydraulic components and systems[M]. Bei Jing: China Machine Press, 2011: 230-231.

[2] Ji Hong, Wang Dongsheng, Liu Xiaoping, Fu Xin. Flow Control Characteristic of the Orifice in Spool Valve with Notches[J]. Transactions of the Chinese Society of Agricultural Engineering, 2009, 40(1): 198-202.

[3] E.Lisowski, G.Filo, J.Rajda. Pressure compensation using flow forces in a multi-section proportional directional control valve[J]. Energy Conversion and Management, 2015, 07: 1052-1064.

[4] Xu Liping, Cui Yanbin, Cai Liujin. Flow Field Simulation and Structure Improvement of the Multi-way Valve in Tractor's Hydraulic System[J]. Chinese Hydraulics \& Pneumatics, 2016(12): 88-93.

[5] Wang Zhaoqiang. Improvement of Valve Port Structure and Performance Analysis for Multiway Valve of Excavator[D]. Lan Zhou: Lanzhou University of Technology, 2010.

[6] Qian feng. Particle swarm optimization and its industrial application[M]. Bei Jing: The Science Publishing Company, 2013.

[7] Li weijia, lan qiuhua, peng yong, etal. Optimization Design of Notches in Spool Valve Based on PSO Algorithm[J]. China Mechanical Engineering, 2015, 26(8): 995-999.

[8] Jiang Tao, Huang Wei, Wang anlin. Topology Design Method for Coupling Valve Throttle Structure of Multi-way Directional Valves[J]. Journal of Xi' an Jiaotong University, 2016, 50(8): 26-31.

[9] Yao Jiye. Spool Valve with Notches simulation based on Fluent[D]. Qin Huang Dao: Yanshan University, 2010. 